\title{
ATP-binding cassette transporters at the zebrafish blood-brain barrier and the potential utility of the zebrafish as an in vivo model
}

\author{
Jordan M. Hotz ${ }^{1}$, Joanna R. Thomas ${ }^{1}$, Emily N. Katz ${ }^{2}$, Robert W. Robey ${ }^{1}$, Sachi Horibata ${ }^{1}$, Michael M. \\ Gottesman $^{1}$ \\ 'Laboratory of Cell Biology, Center for Cancer Research, National Cancer Institute, National Institutes of Health, Bethesda, MD \\ 20892, USA. \\ ${ }^{2}$ Zebrafish Core, Eunice Kennedy Shriver National Institute of Child Health and Human Development, Bethesda, MD 20892, \\ USA.
}

Correspondence to: Dr. Michael M. Gottesman, MD, Chief, Laboratory of Cell Biology, Center for Cancer Research, National Cancer Institute, NIH, 37 Convent Drive, Room 2108, Bethesda, MD 20892, USA. E-mail: mgottesman@nih.gov

How to cite this article: Hotz JM, Thomas JR, Katz EN, Robey RW, Horibata S, Gottesman MM. ATP-binding cassette transporters at the zebrafish blood-brain barrier and the potential utility of the zebrafish as an in vivo model. Cancer Drug Resist 2021;4:620-33. https://dx.doi.org/10.20517/cdr.2021.35

Received: 15 Apr 2021 First Decision: 12 May 2021 Revised: 20 May 2021 Accepted: 25 May 2021 First online: 25 May 2021

Academic Editors: Thomas Litman, Godefridus J. Peters Copy Editor: Xi-Jun Chen Production Editor: Xi-Jun Chen

\begin{abstract}
The brain is protected from toxins by a tightly regulated network of specialized cells, including endothelial cells, pericytes, astrocyes, and neurons, known collectively as the blood-brain barrier (BBB). This selectively permeable barrier permits only the most crucial molecules essential for brain function to enter and employs a number of different mechanisms to prevent the entry of potentially harmful toxins and pathogens. In addition to a physical barrier comprised of endothelial cells that form tight junctions to restrict paracellular transport, there is an active protective mechanism made up of energy-dependent transporters that efflux compounds back into the bloodstream. Two of these ATP-binding cassette $(A B C)$ transporters are highly expressed at the BBB: Pglycoprotein (P-gp, encoded by the $A B C B 1$ gene) and ABCG2 (encoded by the ABCG2 gene). Although a number of in vitro and in vivo systems have been developed to examine the role that $A B C$ transporters play in keeping compounds out of the brain, all have inherent advantages and disadvantages. Zebrafish (Danio rerio) have become a model of interest for studies of the BBB due to the similarities between the zebrafish and mammalian BBB systems. In this review, we discuss what is known about $A B C$ transporters in zebrafish and what information is still needed before the zebrafish can be recommended as a model to elucidate the role of $A B C$ transporters at the BBB.
\end{abstract}

The Author(s) 2021. Open Access This article is licensed under a Creative Commons Attribution 4.0 International License (https://creativecommons.org/licenses/by/4.0/), which permits unrestricted use, sharing, adaptation, distribution and reproduction in any medium or format, for any purpose, even commercially, as long as you give appropriate credit to the original author(s) and the source, provide a link to the Creative Commons license, and indicate if changes were made. 
Keywords: $A B C$ transporters, blood-brain barrier, ABCG2, P-glycoprotein, zebrafish

\section{INTRODUCTION}

\section{The blood-brain barrier}

The blood-brain barrier (BBB) restricts the free exchange of molecules between the blood and the brain and is required to maintain and protect the neural microenvironment. The BBB refers to the specialized adaptations of the microvasculature of the brain. The existence of the BBB was first reported when tracer dyes injected into the circulatory system were not observed to stain the brain ${ }^{[1]}$ and confirmed by the difference in lethality of poisons injected subcutaneously or into the central nervous system $(\mathrm{CNS})^{[2]}$. The cerebral vascular endothelium, along with neurons, pericytes, astrocytes, and the extracellular matrix comprise the basic structural unit of the BBB, termed the neurovascular unit (NVU) ${ }^{[3]}$ [Figure 1]. The intimate physical and chemical links between the cells of the NVU allow for modulation of the BBB in response to stimuli such as inflammation, infection, injury, and neuronal energy requirements ${ }^{[-6-6]}$. Other important components of the brain microenvironment include microglia, macrophages, and fibroblasts ${ }^{[7]}$.

The integrity of the BBB is attributable to key properties of the CNS vascular endothelial cells. Endothelial cells at the mammalian BBB are linked by tight junctions close to their apical membranes that limit paracellular diffusion. Tight junction transmembrane proteins contributing to the BBB are claudin- $1,-3,-5$, -12; occludin, and lipolysis-stimulated protein (LSR ${ }^{[8]}$. Claudin-5 is the most abundant tight junction protein at the $\mathrm{BBB}^{[9]}$. Knockout of claudin- 5 or LSR in mice leads to increased BBB permeability to small molecular weight compounds $s^{[10,1]}$. Occludin is believed to have a regulatory role in tight junctions and is not required for their formation ${ }^{[12]}$. The zonula occludens ( $\left.\mathrm{ZO}\right)$ family are intracellular scaffold proteins that link most tight junction transmembrane proteins to the actin cytoskeleton, stabilizing these cell-cell adhesions $^{[8]}$. ZO-1 and ZO-2 are expressed in endothelial cells of the BBB, whereas ZO- 3 is not ${ }^{[13]}$. ZO-1 and ZO-2 have functional redundancy in epithelial cells, and deletion of both results in a complete lack of tight junctions ${ }^{[14]}$. In human-derived microvascular endothelial cells, however, ZO-1 is required for tight junction formation and structural integrity, independently of $\mathrm{ZO}-2^{[15]}$. Endothelial cells at the $\mathrm{BBB}$ also have low levels of micropinocytosis in comparison to peripheral endothelial cells, limiting transcytosis ${ }^{[16,17]}$.

The remaining cell types that form the canonical NVU are pericytes and astrocytes. Pericytes are mural cells that partially cover the ablumenal endothelial walls of microvessels and are embedded in the vascular basement membrane. They form cell-cell contacts with endothelial cells and astrocytic end-feet ${ }^{[18]}$. Pericytes are required for the formation of the $\mathrm{BBB}^{[19]}$ and for maintenance of $\mathrm{BBB}$ function in adulthood ${ }^{[20]}$. Astrocytes, a type of glial cells that make up a significant portion of the CNS, form contacts with both microvessel walls and neurons, providing a cellular link between neurons and vasculature ${ }^{[21]}$. However, astrocytes are not required in the formation of the $\mathrm{BBB}$, as a functional $\mathrm{BBB}$ is present in rodent embryogenesis prior to astrocyte differentiation ${ }^{[19]}$. Astrocytes are important in the modulation and maintenance of the BBB, and are able to induce the formation of barrier-like properties in non-CNSderived endothelial cells, as demonstrated by transplant studies ${ }^{[22]}$ and co-culture experiments ${ }^{[2,23]}$.

CNS endothelial cells express two types of transporters: nutrient transporters and efflux transporters ${ }^{[18]}$. Nutrient transporters allow the entry of key nutrients, such as glucose and insulin, into the brain ${ }^{[24]}$. The efflux transporters expressed belong to the adenosine triphosphate (ATP) binding cassette (ABC) transporter family, which are encoded by a diverse family of 48 human genes. Some of these transporters have specific physiological substrates, whereas others can transport a broad range of exogenous compounds $^{[25]}$. ABC transporters form a selective, active barrier protecting the brain from toxins, 


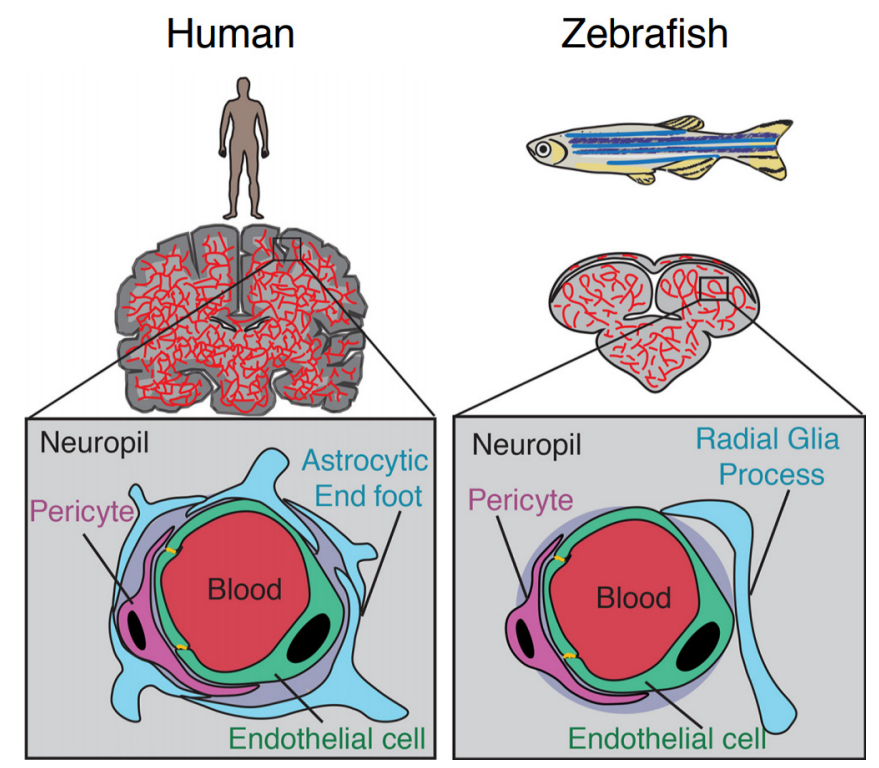

Figure 1. Comparison of the human and zebrafish neurovascular unit. Adapted from O'Brown et al. ${ }^{[41]}$.

xenobiotics, and a wide range of drugs ${ }^{[26]}$. Two of the most highly expressed $\mathrm{ABC}$ transporters at the $\mathrm{BBB}$ are P-glycoprotein (P-gp, encoded by the $A B C B 1$ gene) and ABCG2 (encoded by the ABCG2 gene) ${ }^{[27]}$. Throughout this review, we use the terms P-gp and ABCG2 to refer to the human proteins. ABCC family members such as $\mathrm{ABCC} 2, \mathrm{ABCC} 4$ and $\mathrm{ABCC} 5$ are also expressed on the lumenal surface of brain endothelial cells ${ }^{[26]}$. The significant role of P-gp at the BBB was demonstrated in 1994 by Schinkel et al. ${ }^{[28]}$, who found that deletion of the Abcb1a gene in mice caused a lethal 90-fold increase in brain penetration of the drug ivermectin that is used as an anti-parasitic. Deletion of the $A b c g 2$ gene in mice led to increased phototoxicity due to high blood levels of a chlorophyll catabolite ${ }^{[29]}$. Mice deficient in both murine homologs of human $A B C B 1, A b c b 1 a$ and $A b c b 1 b$, as well as the murine $A b c g 2$ gene have shown that both proteins work cooperatively to prevent entry of many chemotherapeutic agents into the brain ${ }^{[25]}$. Toxicity from ivermectin, used commonly to prevent and treat heartworm in dogs, occurs in American collies due to loss of expression of canine P-gp ${ }^{[30]}$ and humans lacking functional P-gp cannot tolerate ivermectin ${ }^{[31]}$. The multidrug efflux transporters, P-gp and ABCG2, both have wide substrate specificities and are therefore well-suited to protect the CNS from a wide range of toxins ${ }^{[26]}$.

The BBB is highly efficient at protecting the brain, with the impermeability and selectivity of the barrier resulting in exclusion of all large-molecule neurotherapeutics, and $98 \%$ of small molecule drugs ${ }^{[32]}$. The delivery of small molecule drugs into the brain for treatment of CNS-related diseases and brain cancers represents a significant challenge in drug development. $\mathrm{ABC}$ transporter expression at the BBB plays a pivotal role in preventing the brain penetration by these therapeutics. As a better understanding of the role of $\mathrm{ABC}$ transporters at the $\mathrm{BBB}$ could lead to the development of CNS-targeted therapeutics, several in vitro and in vivo models have been developed to facilitate research in this area.

\section{Models for studying ABC transporters at the BBB}

In vitro and in vivo model systems have been used to explore the contributions of $\mathrm{ABC}$ transporters to the integrity of the BBB. Some of the more complex in vitro models permit visualization of the structure and function of the NVU cells, while in vivo models reveal gene expression patterns and membrane permeability through real-time imaging ${ }^{[3,34]}$. Identifying optimal models would aid in the development of effective therapeutic agents that can bypass the $\mathrm{BBB}$ and target specific neurological disease sites. 
Transwell assays

Endothelial cell lines that form polarized monolayers and tight junctions were some of the first models used to examine the role transporters play in keeping substrates out of the brain. Transwell assays using MadinDarby canine kidney (MDCK) or pig kidney (LLC-PK1) cells transfected with P-gp have routinely been used to identify substrates of P-gp. MDCK and LLC-PK1 cells have also been transfected to express both Pgp and ABCG2 to explore the effects of their coexpression ${ }^{[35-37]}$. Caco- 2 colorectal adenocarcinoma cells also form tight junctions and express relatively high endogenous levels of P-gp and ABCG2. However, transwell assays are over-simplistic models that do not address the potential contributions of other cell types or blood flow in determining BBB permeability. Immortalized brain endothelial cell lines of human origin, such as the hCMEC/D3 line, or those of non-human origin, such as the murine cell lines MBEC4 and bEND.3, have also been used in transwell assays. However, these models have poor barrier properties, resulting in low levels of transendothelial electrical resistance and a poor BBB phenotype with weaker tight junction formation ${ }^{[38]}$. Additionally, transporter expression is much lower in the immortalized lines compared to primary cells.

\section{Co-culture models}

Neurovascular cell co-cultures are more advantageous models than monocultures of isolated brain endothelial cells because brain endothelial cells lose their BBB properties when they are removed from the brain microenvironment ${ }^{[33]}$. Combining endothelial cells with pericytes and astrocytes in a culture system stimulates the re-creation of the NVU environmental conditions for more accurate experimentation ${ }^{[39]}$. Such co-cultures simulate the BBB environment by inducing endothelial cell differentiation as well as by including $\mathrm{BBB}$ specific-tight junction proteins and $\mathrm{ABC}$ transporters ${ }^{[3,34,40]}$. Further developments have led to BBB-on-a-chip models, in vitro models that produce sheer stress for in vivo - like results concerning junction tightness and biomarker permeability ${ }^{[39]}$. Although the BBB-on-a-chip has overcome some limitations of the traditional transwell culture model, it is a flexible technology that can cause different forms of on-chip models to vary in microchannel size, cell type, and permeability measurement, which can lead to discrepancies in experimental results ${ }^{[39]}$.

\section{Mouse models}

The mouse is the most common species used for modeling the human BBB due to its similar vasculature and cell composition ${ }^{[34,41]}$. Knockout mouse models have been instrumental in demonstrating the protective role that transporters play at the $\mathrm{BBB}$. As mentioned previously, mice that lack both murine P-gp homologs (Abcb1a and Abcb1b) as well as murine Abcg2 have been used to show that many chemotherapeutic agents do not enter the brain due to the expression of these transporters ${ }^{[25]}$. In one example, brain penetration of the anaplastic lymphoma kinase inhibitor, brigatinib, was approximately 42 -fold higher in mice lacking $\mathrm{Abcb} 1 \mathrm{a} / \mathrm{Abcb} 1 \mathrm{~b} / \mathrm{Abcg} 2$ compared to wild-type mice ${ }^{[42]}$. While mice are frequently used as models to study the BBB, they are expensive and not amenable to high throughput analysis ${ }^{[34,43]}$.

Even with the improvement over time of both in vitro and in vivo models that mimic the BBB environment and physiology, there are still certain disadvantages of using these models. Overcoming the disadvantages associated with the current $\mathrm{BBB}$ models used to study the role of $\mathrm{ABC}$ transporters may advance drug development and treatment of neurological diseases. The zebrafish has several advantages over other in vivo models of the $\mathrm{BBB}$ and has been suggested as a model for examining the role of $\mathrm{ABC}$ transporters expressed at the brain endothelium. 


\section{THE ZEBRAFISH AS A MODEL TO STUDY ABC TRANSPORTERS AT THE BBB}

Zebrafish possess many characteristics that make them a suitable model for studying the BBB. They are small, tropical, fresh-water teleost fish growing only to about $3 \mathrm{~cm}$ in length as adults, and can be housed in fairly small aquaria. Zebrafish require much less maintenance than mice. After external egg fertilization, their embryos are transparent and develop rapidly, making them ideal for observing biological processes in vivo. Large numbers of embryos are produced, providing the opportunity for large-scale high-throughput studies. Small-molecule screens have identified compounds that inhibit biological processes, demonstrating the usefulness of zebrafish in the drug discovery process for human disease ${ }^{[44]}$. Embryonic zebrafish can be imaged easily on a wide range of microscopes, with potential for sub-cellular and super-resolution, especially when combined with post-fixation processing techniques such as a tissue clearance ${ }^{[45]}$ and expansion microscopy ${ }^{[46]}$. Many useful fluorescent transgenic lines have been created with cell-specific promoters, such as the glut1b:mCherry line, with specifically labeled brain endothelial cells ${ }^{[47]}$.

\section{Similarities between the zebrafish and human BBB}

Zebrafish have a BBB that is structurally and functionally similar to that of mammals. Permeability studies with dyes and tracers, such as FITC-dextran and HRP, have demonstrated the presence of a functional BBB in both developing and adult zebrafish ${ }^{[48-50]}$. The acquisition of BBB properties occurs in brain endothelial cells during angiogenesis starting at $30 \mathrm{~h}$ post-fertilization (hpf $)^{[47,51]}$, and the BBB continues to mature, with increasing restriction of lower molecular weight substances with age, assessed up to 10 days postfertilization $(\mathrm{dpf})^{[49]}$.

The basic structure of the NVU is similar to that of humans and mice, with the notable exception of a direct equivalent to astrocytes [Figure 1] ${ }^{[41]}$. Zebrafish brain endothelial cells have many of the same adaptations as their mammalian counterparts. Teleost fish have homologs for the majority of the claudin family of transmembrane tight junction proteins, including claudin- $5^{[52]}$. Zebrafish also have homologs from the ZO protein family, such as ZO-1 [tight junction protein (tjp) $1 a$ and tjp 1 b], ZO-2 (tjp2a and tjp2b), and ZO-3 ( tjp3). Tjp $1 b$ is the dominant gene expressed in the zebrafish brain ${ }^{[53]}$, suggesting commonality with mammalian BBB tight junctions for which ZO-1 is crucial ${ }^{[15]}$. Zebrafish express homologs of claudin-5 and ZO-1, which are essential to the integrity of the mammalian BBB in brain microvessels, where they are localized to endothelial cell-cell junctions ${ }^{[48,51]}$. Physical evidence of tight junctions between endothelial cells of the $\mathrm{BBB}$ has been observed at $3 \mathrm{dpf}^{[54]}$ and $10 \mathrm{dpf}$ by electron microscopy ${ }^{[49]}$.

Reduced transcytosis across endothelial cells is another important adaptation of the mammalian BBB. During early zebrafish development from 3-10 dpf, suppression of endothelial cell transcytosis correlates with a decrease in BBB permeability ${ }^{[54]}$. The mammalian transcytosis regulator protein, MFSD2A, functions to reduce transcytosis in murine CNS endothelial cells ${ }^{[5]}$, and has a conserved function in zebrafish. Zebrafish with morpholino knockdown or mutation of the zebrafish homolog, $m f s d 2 a a$, leads to increased BBB permeability ${ }^{[54,56]}$. Similar to humans, zebrafish CNS endothelial cells also express transporters that contribute to the functionality of the BBB, including nutrient transporters, such as Glut $1^{[47]}$, and multidrug efflux transporters, which are discussed below.

In the mammalian $\mathrm{BBB}$, pericytes are embedded in the vascular basement membrane in close proximity to endothelial cells, and are believed to play an important role in the formation and maintenance of the $\mathrm{BBB}^{[18]}$. Pericytes have the same localization in the zebrafish BBB, as evidence by electron microscopy at 3 dpf indicates ${ }^{[54]}$. Pericytes are associated with brain microvessels from $48 \mathrm{hpf}$, after which they continue to proliferate and migrate to cover the vascular endothelium until $5 \mathrm{dpf}^{[57,58]}$. Zebrafish embryos with a missense mutation in notch 3 have a deficiency in pericyte cell numbers due to reduced proliferation of 
pericyte progenitor ${ }^{[57]}$. These mutant embryos have brain hemorrhages and a more permeable BBB, with an absence of endothelial or tight junction defects ${ }^{[57]}$, similar to mammals lacking pericytes ${ }^{[20]}$. A panel of mammalian pericyte marker genes were used in order to identify brain pericytes in zebrafish. Zebrafish pericytes expressed both $p d g f r b$, a PDFRb homolog, and tagln2, a smooth muscle $\alpha$-actin homolog, but not rgs5a, desmin $a$, desmin $b$, or the NG2 homolog, cspg $4^{[57]}$. Zebrafish pericytes appear to have both neural crest and mesoderm origin ${ }^{[58]}$, whereas mammalian pericytes develop only from the neural crest ${ }^{[59]}$. The exact role of zebrafish brain pericytes and the consequences of the differences between expression profiles and origins of zebrafish and mammalian pericytes remain unclear.

Zebrafish lack a direct equivalent of classic stellate mammalian astrocytes; however, Chen et al. ${ }^{[60]}$ recently identified a population of cells derived from radial glial cells that are remarkably similar to astrocytes and could potentially be considered an astrocytic equivalent. Through evolution, glia have diversified to include radial glia, astrocytes, microglia, oligodendrocytes, and ependymal cells; each with different functions in the brain $^{[61]}$. In mammals, radial glial cells differentiate to give rise to both neurons and astrocytes ${ }^{[62]}$. Zebrafish radial glia and mature mammalian astrocytes share the expression of many proteins, such as glial fibrillary acidic protein (GFAP), glutamate aspartate transporter, and aquaporin-4, implying a molecular similarity ${ }^{[60,63]}$.

Astrocyte function depends on highly branched morphology, which allows contact with synapses, neurons, the vasculature, and other glial cells. Radial glial cells, in contrast, have a much simpler morphology, with fewer and longer processes than astrocytes ${ }^{[61]}$. The astrocyte-like population of radial glia, identified by Chen et al. ${ }^{[60]}$, have a complex branched morphology that is very similar to mammalian astrocytes. These radial glial cells expressing GFAP have processes that make contact with neurons, synapses ${ }^{[60]}$, and microvasculature ${ }^{[48]}$, demonstrating a similar spatial organization to mammalian astrocytes ${ }^{[61]}$. Functional conservation exists between mammalian astrocytes and zebrafish astrocyte-like radial glia. For example, both exhibit spontaneous calcium transients ${ }^{[0,64]}$ and respond to damage of the $\mathrm{CNS}^{[65]}$. Controversy remains in the field as to whether radial glial cells are a rudimentary equivalent of astrocytes in zebrafish, or if the identified population of more complex radial glial cells are in fact bona fide astrocytes. Regardless of the consensus, the function of these cells with regards to the BBB has yet to be determined.

\section{Zebrafish $A B C$ transporters at the $B B B$}

Zebrafish have two homologs of human $A B C B 1$. These two genes are currently named $a b c b 4$ and $a b c b 5$. They were previously designated as $a b c b 1 b$ and $a b c b 1 a$, respectively, but were later renamed based on chromosomal localization ${ }^{[66]}$. The zebrafish Abcb4 protein has 63\% amino acid similarity with human P-gp, while Abcb5 has 57\% similarity ${ }^{[49]}$. Earlier studies showed that the $\mathrm{C} 219$ antibody, developed to detect human P-gp ${ }^{[6]]}$, reacted with a protein found in zebrafish brain endothelial cells, likely due to preservation of the $\mathrm{C} 219$ epitope in zebrafish Abcb4 and $\mathrm{Abcb} 5^{[44,49]}$. In our own studies, we have also noted $\mathrm{C} 219$ antibody reactivity in the vasculature of the zebrafish brain [Figure 2]. A transporter at the zebrafish BBB similar in function to P-gp has been demonstrated by functional assays with rhodamine 123, a known P-gp substrate. After injecting embryos at 3, 5, 8, and $10 \mathrm{dpf}$ with rhodamine 123 . Fleming et al. ${ }^{[49]}$ found that rhodamine was actively effluxed out of the brain at $8 \mathrm{dpf}$. In another study, rhodamine 123 was injected into the circulation of zebrafish embryos at 3, 4, 5, 6, and $7 \mathrm{dpf}$ and brain fluorescence was monitored. At the earlier time points, dye was retained in the brain region, indicating a lack of transporter functionality. In the 6 and $7 \mathrm{dpf}$ embryos, no fluorescence was observed, indicating that a homologous transporter was preventing brain penetration ${ }^{[68]}$. However, it is unclear whether one or both of the P-gp homologs are expressed at the zebrafish $\mathrm{BBB}$ and it is not known whether the substrate specificity of the transporters is comparable to that of P-gp. Known inhibitors of human P-gp have been used to demonstrate that a P-gp homolog contributes to the BBB integrity of other teleost fish, including killifish, rainbow trout, and dogfish sharks ${ }^{[69-72]}$. 


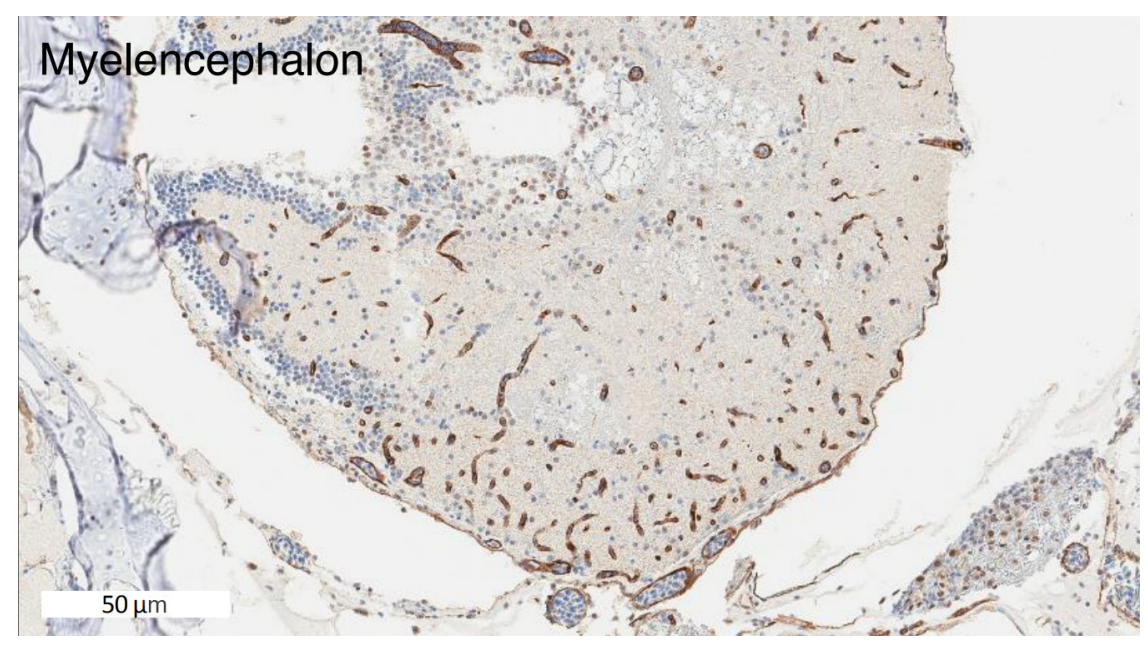

Figure 2. The C219 antibody reacts with cells in the zebrafish brain. Formalin-fixed, paraffin-embedded whole adult zebrafish were stained with the C219 antibody. Positive staining is observed in what appears to be the vasculature and is consistent with localization to the blood-brain-barrier.

For $A B C G 2$, four homologs in the zebrafish have been identified: $\operatorname{abcg} 2 a, a b c g 2 b, a b c g 2 c$, and $a b c g 2 d^{[73]}$. Comparison of the amino acid sequence of the zebrafish homologs with human ABCG2 revealed Abcg2a to have the highest amino acid sequence identity at $60 \%$, followed by Abcg2d (48\%), Abcg2c (45\%), and Abcg2b $(44 \%)^{[74]}$. Based on synteny analyses, abcg2d is the closest homolog of human $A B C G 2^{[71]}$. Although it is reasonable to assume that one, or possibly more, of the zebrafish ABCG2 homologs is or are expressed at the $\mathrm{BBB}$, to date, no studies have localized any of them to the brain vasculature. Cells transfected to overexpress zebrafish Abcg2a were found to transport Hoechst 33342, but this appears to be the only known substrate of the zebrafish ABCG2 homologs ${ }^{[74]}$. Further studies are needed to confirm the existence of an ABCG2 homolog, or homologs at the zebrafish BBB and to determine the substrate specificity of the proteins.

Zebrafish also express homologs of human $A B C C 2, A C B C C 4$ and $A B C C 5$, which are localized to brain endothelial cell lumenal membranes in mammalian systems. Zebrafish express $A B C C 4$ and $A B C C 5$ in the brain, although localization by cell type has not been determined ${ }^{[55,76]}$. There is no evidence yet for expression of $A B C C 2$ in the zebrafish brain. However, it is expressed and is functional in isolated brain capillaries of killifish, which are also teleost fish ${ }^{[72]}$.

\section{Potential problems with the zebrafish model}

Because of the noted similarities between the zebrafish and the mammalian BBB, the zebrafish has been suggested as a good model to study the role of transporters at the $\mathrm{BBB}^{[43]}$. In one comprehensive study, Kim et al. ${ }^{[77]}$ compared the zebrafish brain penetration of several compounds to penetration of the mouse brain. Among the compounds tested was loperamide, an analog of which has been explored for use as a PET imaging agent to measure P-gp function at the $\mathrm{BBB}^{[78]}$. The estimated partition coefficient for loperamide after oral administration in the zebrafish was found to increase by about 2 -fold when coadministered with the P-gp inhibitor tariquidar, which was comparable to the 4-fold increase observed in mice. For several of the compounds tested, including both compounds known to cross the BBB and those that do not, the partition coefficients were similar in zebrafish and mice. However, for a subset of unknown compounds unique to the study, the partition coefficients for the zebrafish did not correlate with those of mice. As suggested by the authors, differences in influx or efflux transporters could be the reason for the discrepancy. 
Indeed, this is one of the problems with suggesting that the zebrafish could be used to model transporter activity at the BBB. The assumption is that zebrafish transporters function much like human transporters. However, there is a paucity of data with regard to the substrate specificity of homologous zebrafish transporters. Fischer et al. ${ }^{[6]]}$ examined the accumulation of P-gp substrates rhodamine B and calcein-AM in whole embryo assays and demonstrated increased fluorescence in the embryos treated with the substrates and P-gp inhibitors compared to the embryos treated with the substrates alone. In a subsequent study, a number of compounds were also found to increase rhodamine B fluorescence in embryos at $72 \mathrm{hpf}$, potentially due to inhibition of zebrafish Abcb $4^{[79]}$. The cyanobacterial toxin microcystin-LR was also found to be a substrate of Abcb4 based on cytotoxicity assays with LLC-PK1 cells transfected with zebrafish abcb4 as well as microinjection of zebrafish embryos with $a b c b 4^{[80]}$. Expression of $A b c b 5$ was recently localized to zebrafish ionocytes and the fluorescent P-gp substrates calcein-AM and DiOC6 were transported, demonstrating that Abcb4 and Abcb5 can efflux known P-gp substrates. These few studies report what little is known about the substrate specificities of the zebrafish transporters that are homologous to P-gp.

To determine the substrate specificity of Abcb4 and Abcb5, we transfected HEK293 cells with empty vector (Vector) or with vectors encoding human $A B C B 1$ (MDR-19), zebrafish abcb4 (ZF Abcb4), or zebrafish abcb5 (ZF Abcb5). We then performed cytotoxicity assays with the known human P-gp substrates etoposide, doxorubicin, and mitoxantrone. As shown in Figure 3A, zebrafish Abcb4, zebrafish Abcb5, and P-gp conferred comparable levels of resistance to etoposide, while zebrafish Abcb5 conferred much less resistance to doxorubicin and mitoxantrone, suggesting that the substrate specificity profile of zebrafish Abcb5 might be narrower compared to zebrafish Abcb4 and P-gp. Further evidence was garnered from flow cytometry assays with fluorescent substrates [Figure 3B]. When we incubated MDR-19, ZF Abcb4, and ZF Abcb5 with the fluorescent substrate BODIPY-ethylenediamine (EDA) in the presence or absence of the Pgp inhibitor valspodar, we noted decreased intracellular fluorescence of BODIPY-EDA in the MDR-19 and $\mathrm{ZF}$ Abcb4 cells that was reversed by the addition of valspodar. However, transport was not observed in the ZF Abcb5 cells. Since these results pointed to a difference in substrate specificity between the zebrafish homologs, we performed a high-throughput screen on the transfected cells using 90 substrates of $\mathrm{P}$-gp ${ }^{[81]}$. By comparing the drug response area under the curve for cells transfected with empty vector and the different transporters, we found that the substrate specificity profile of zebrafish Abcb4 is similar to that of human P$\mathrm{gp}^{[82]}$. Additionally, we found that the efficacy of human P-gp inhibitors varied for zebrafish Abcb4 and Abcb5 and depended on the substrate examined. Using RNAScope in situ probes, we noted that zebrafish $a b c b 4$ colocalized with C219 staining in the zebrafish brain vasculature. Vessels in the zebrafish brain that were claudin-5 positive also stained positively with $a b c b 4$ probes $^{[82]}$. These results support the hypothesis that zebrafish Abcb4 is the functional homolog of human P-gp at the zebrafish BBB.

With regard to ABCG2, even less is known about transport substrates, with Hoechst 33342 being the only confirmed substrate for zebrafish Abcg2 $\mathrm{a}^{[74]}$. In preliminary studies with HEK293 cells transfected with empty vector (Vector), or with vectors encoding human ABCG2 (R5), zebrafish abcg2a (ZF Abcg2a), zebrafish $a b c g 2 b$ (ZF Abcg2b), zebrafish abcg2c (ZF Abcg2c), or zebrafish abcg2d (ZF Abcg2d), we noted very different substrate specificities in the zebrafish homologs as compared to human ABCG2. As shown in Figure 4, all of the zebrafish forms conferred similar levels of resistance to the known ABCG2 substrates THZ531, THZ1 ${ }^{[83]}$, and gedatolisib ${ }^{[81]}$. However, stark differences were noted for mitoxantrone, SN-38 and topotecan, as only Abcg2a and Abcg2d conferred appreciable resistance to mitoxantrone or SN-38, and none of the zebrafish homologs conferred resistance to topotecan. Thus, none of the zebrafish homologs can be considered functionally equivalent to human ABCG2 based on our preliminary data, although further confirmation is necessary. 
A
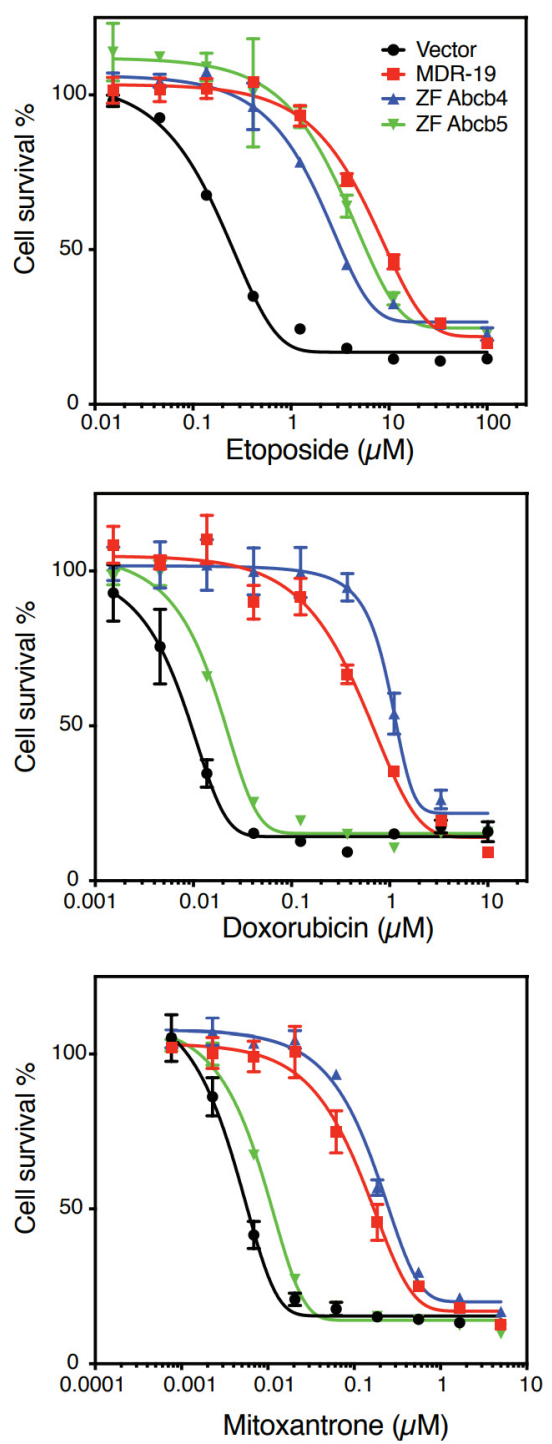

$\mathrm{B}$
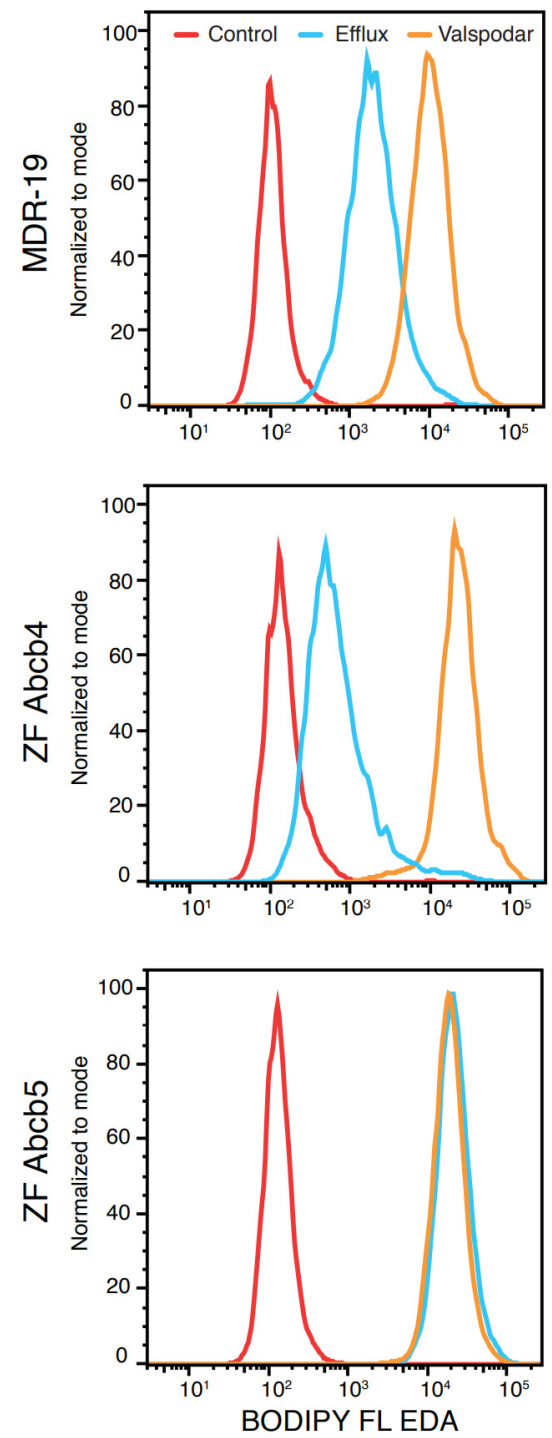

Figure 3. Comparison of the substrate specificity of human P-gp and zebrafish homologs. (A) Three-day cytotoxicity assays were performed with etoposide, doxorubicin or mitoxantrone on empty-vector-transfected HEK293 cells (Vector), HEK293 cells that express human P-gp (MDR-19), zebrafish Abcb4 (ZF Abcb4), or zebrafish Abcb5 (ZF Abcb5). (B) MDR-19, ZF Abcb4 and ZF Abcb5 cells were incubated with $500 \mathrm{nM}$ BODIPY-ethylenediamine (EDA) in the presence or absence of $10 \mu \mathrm{M}$ valspodar for 30 min. The medium was subsequently removed and replaced with substrate-free medium continuing without or with valspodar for another $1 \mathrm{~h}$, after which intracellular fluorescence was measured on a flow cytometer.

Further clarification is also needed with regard to when $\mathrm{ABC}$ transporters are expressed at zebrafish brain endothelial cells. Fleming et al. ${ }^{[49]}$ demonstrated colocalization of the $\mathrm{C} 219$ antibody within the vasculature of $8 \mathrm{dpf}$ embryos, but not in those of $3 \mathrm{dpf}$ embryos. Similarly, they noted exclusion of rhodamine 123 from the brain in 8 and $10 \mathrm{dpf}$ embryos, but not in $5 \mathrm{dpf}$ embryos. However, it is not yet clear if a zebrafish P-gp homolog is localized to the vasculature between 5 and $8 \mathrm{dpf}$. Additionally, no data are available with regard to ABCG2 homologs, so it is not clear if ABCG2 colocalizes at the same time as the P-gp homolog or if it follows a different timeline. Knowing the timepoint of $\mathrm{BBB}$ closure in zebrafish is important before proceeding with experimentation, especially for potential translational studies. 

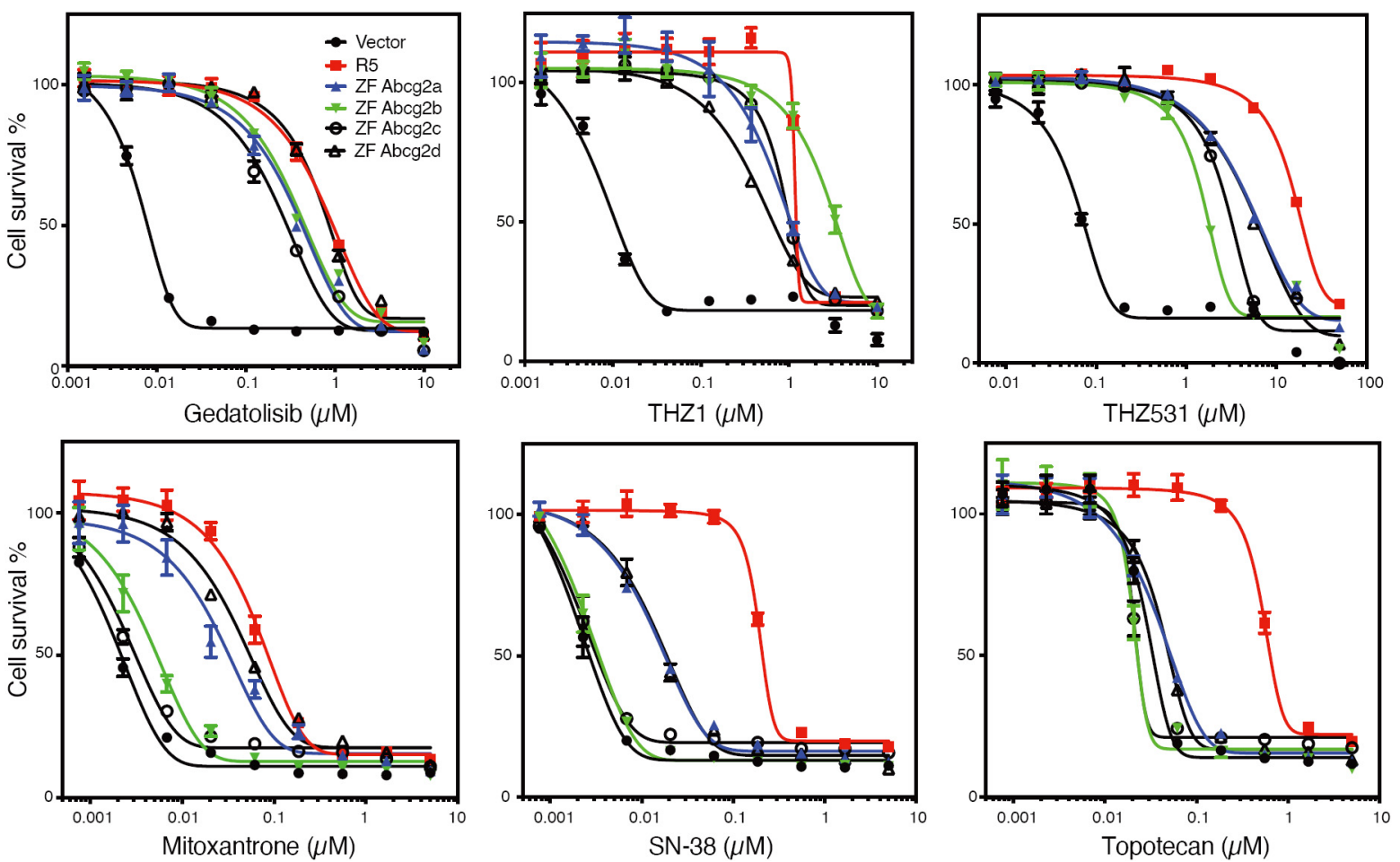

Figure 4. Zebrafish $A B C G 2$ homologs do not functionally mimic human $A B C G 2$. Three-day cytotoxicity assays were performed with gedatolisib, THZ1, THZ531, mitoxantrone, SN-38, or topotecan on empty-vector-transfected HEK293 cells (Vector), or HEK293 cells that express human ABCG2 (R5), zebrafish Abcg2a (ZF Abcg2a), Abcg2b (ZF Abcg2b), Abcg2c (ZF Abcg2c), or zebrafish Abcg2d (ZF Abcg2d).

\section{CONCLUSIONS}

Due to the highly conserved nature of zebrafish and mammalian BBB systems, as well as the small size and relatively ease of care of the zebrafish, it has emerged as a potentially useful model system for the study of $\mathrm{ABC}$ transporters at the $\mathrm{BBB}$. Additionally, the ease of manipulating zebrafish genetics may lead to discoveries concerning maintenance of BBB integrity. Although zebrafish Abcb4 does appear to functionally copy P-gp at the zebrafish BBB, significant questions remain in terms of the substrate specificity of the ABCG2 homologs and their localization. If preliminary data gleaned from cell line models hold true, genetic modifications leading to humanization of the transporters could be possible; mice expressing human $A B C B 1$ have already been generated ${ }^{[84]}$. In any case, we anticipate that zebrafish will play a role in the development of therapeutic agents that can circumvent $\mathrm{ABC}$ transporters at the $\mathrm{BBB}$, allowing improved brain penetration and more effective treatment.

\section{DECLARATIONS}

\section{Acknowledgements}

The authors would like to thank Andrew Warner, Donna Butcher, Jennifer Matta, and Elijah Edmondson of the Pathology/Histotechnology Laboratory at the NCI at Frederick for performing immunohistochemical studies on zebrafish. Additionally, we are grateful to George Leiman for editorial assistance. This research was supported by the Intramural Research Program of the National Cancer Institute. The content of this publication does not necessarily reflect the views or policies of the Department of Health and Human Services, nor does mention of trade names, commercial products, or organizations imply endorsement by the United States Government. 


\section{Authors' contributions}

Writing of the review: Hotz JM, Thomas JR, Katz EN, Robey RW, Horibata S, Gottesman MM

Availability of data and materials

Not applicable.

\section{Financial support and sponsorship}

This publication was funded by the Intramural Research Program of the National Institutes of Health, the National Cancer Institute.

\section{Conflicts of interest}

All authors declare that there are no conflicts of interest.

\section{Ethical approval and consent to participate}

Not applicable.

\section{Consent for publication}

Not applicable.

\section{Copyright}

The Author(s) 2021.

\section{REFERENCES}

1. Ehrlich P. Das Sauerstoff-Bedurfnis des Organismus: eine farbenanalytische Studie. Berlin: Hirschward; 1885.

2. Lewandowsky M. Zur Lehre der Zerebrospinalflussigkeit. Z Klin Med 1900;40:480-4.

3. Hawkins BT, Davis TP. The blood-brain barrier/neurovascular unit in health and disease. Pharmacol Rev 2005;57:173-85. DOI PubMed

4. Blanchette M, Daneman R. Formation and maintenance of the BBB. Mech Dev 2015;138 Pt 1:8-16. DOI PubMed

5. Muoio V, Persson PB, Sendeski MM. The neurovascular unit - concept review. Acta Physiol (Oxf) 2014;210:790-8. DOI PubMed

6. Neuwelt EA. Mechanisms of disease: the blood-brain barrier. Neurosurgery 2004;54:131-40; discussion 41. DOI PubMed

7. Zhao X, Chen R, Liu M, Feng J, Chen J, Hu K. Remodeling the blood-brain barrier microenvironment by natural products for brain tumor therapy. Acta Pharm Sin B 2017;7:541-53. DOI PubMed PMC

8. Greene C, Hanley N, Campbell M. Claudin-5: gatekeeper of neurological function. Fluids Barriers CNS 2019;16:3. DOI PubMed PMC

9. Ohtsuki S, Yamaguchi H, Katsukura Y, Asashima T, Terasaki T. mRNA expression levels of tight junction protein genes in mouse brain capillary endothelial cells highly purified by magnetic cell sorting. J Neurochem 2008;104:147-54. DOI PubMed

10. Sohet F, Lin C, Munji RN, et al. LSR/angulin-1 is a tricellular tight junction protein involved in blood-brain barrier formation. $J$ Cell Biol 2015;208:703-11. DOI PubMed PMC

11. Nitta T, Hata M, Gotoh S, et al. Size-selective loosening of the blood-brain barrier in claudin-5-deficient mice. $J$ Cell Biol 2003;161:653-60. DOI PubMed PMC

12. Saitou M, Furuse M, Sasaki H, et al. Complex phenotype of mice lacking occludin, a component of tight junction strands. Mol Biol Cell 2000;11:4131-42. DOI PubMed PMC

13. Inoko A, Itoh M, Tamura A, Matsuda M, Furuse M, Tsukita S. Expression and distribution of ZO-3, a tight junction MAGUK protein, in mouse tissues. Genes Cells 2003;8:837-45. DOI PubMed

14. Umeda K, Ikenouchi J, Katahira-Tayama S, et al. ZO-1 and ZO-2 independently determine where claudins are polymerized in tightjunction strand formation. Cell 2006;126:741-54. DOI PubMed

15. Tornavaca O, Chia M, Dufton N, et al. ZO-1 controls endothelial adherens junctions, cell-cell tension, angiogenesis, and barrier formation. J Cell Biol 2015;208:821-38. DOI PubMed PMC

16. Reese TS, Karnovsky MJ. Fine structural localization of a blood-brain barrier to exogenous peroxidase. J Cell Biol 1967;34:207-17. DOI PubMed PMC

17. Chow BW, Gu C. Gradual suppression of transcytosis governs functional blood-retinal barrier formation. Neuron 2017;93:1325-33.e3. DOI PubMed PMC

18. Daneman R, Prat A. The blood-brain barrier. Cold Spring Harb Perspect Biol 2015;7:a020412. DOI PubMed PMC

19. Daneman R, Zhou L, Kebede AA, Barres BA. Pericytes are required for blood-brain barrier integrity during embryogenesis. Nature 2010;468:562-6. DOI PubMed PMC

20. Armulik A, Genové G, Mäe M, et al. Pericytes regulate the blood-brain barrier. Nature 2010;468:557-61. DOI PubMed 
21. Abbott NJ, Rönnbäck L, Hansson E. Astrocyte-endothelial interactions at the blood-brain barrier. Nat Rev Neurosci 2006;7:41-53. DOI PubMed

22. Janzer RC, Raff MC. Astrocytes induce blood-brain barrier properties in endothelial cells. Nature 1987;325:253-7. DOI PubMed

23. Igarashi Y, Utsumi H, Chiba H, et al. Glial cell line-derived neurotrophic factor induces barrier function of endothelial cells forming the blood-brain barrier. Biochem Biophys Res Commun 1999;261:108-12. DOI PubMed

24. Fu BM. Transport across the blood-brain barrier. Adv Exp Med Biol 2018;1097:235-59. DOI PubMed

25. Robey RW, Pluchino KM, Hall MD, Fojo AT, Bates SE, Gottesman MM. Revisiting the role of ABC transporters in multidrugresistant cancer. Nat Rev Cancer 2018;18:452-64. DOI PubMed PMC

26. Hartz AM, Bauer B. ABC transporters in the CNS - an inventory. Curr Pharm Biotechnol 2011;12:656-73. DOI PubMed

27. Schinkel AH, Jonker JW. Mammalian drug efflux transporters of the ATP binding cassette (ABC) family: an overview. Adv Drug Deliv Rev 2003;55:3-29. DOI PubMed

28. Schinkel AH, Smit JJ, van Tellingen O, et al. Disruption of the mouse mdrla P-glycoprotein gene leads to a deficiency in the bloodbrain barrier and to increased sensitivity to drugs. Cell 1994;77:491-502. DOI PubMed

29. Jonker JW, Buitelaar M, Wagenaar E, et al. The breast cancer resistance protein protects against a major chlorophyll-derived dietary phototoxin and protoporphyria. Proc Natl Acad Sci U S A 2002;99:15649-54. DOI PubMed PMC

30. Mealey KL, Bentjen SA, Gay JM, Cantor GH. Ivermectin sensitivity in collies is associated with a deletion mutation of the mdr1 gene. Pharmacogenetics 2001;11:727-33. DOI PubMed

31. Baudou E, Lespine A, Durrieu G, et al. Serious ivermectin toxicity and human ABCB1 nonsense mutations. N Engl J Med 2020;383:787-9. DOI PubMed

32. Pardridge WM. The blood-brain barrier: bottleneck in brain drug development. NeuroRx 2005;2:3-14. DOI PubMed PMC

33. Canfield SG, Stebbins MJ, Morales BS, et al. An isogenic blood-brain barrier model comprising brain endothelial cells, astrocytes, and neurons derived from human induced pluripotent stem cells. J Neurochem 2017;140:874-88. DOI PubMed PMC

34. Jackson S, Meeks C, Vezina A, Robey RW, Tanner K, Gottesman MM. Model systems for studying the blood-brain barrier: Applications and challenges. Biomaterials 2019;214:119217. DOI PubMed

35. Poller B, Wagenaar E, Tang SC, Schinkel AH. Double-transduced MDCKII cells to study human P-glycoprotein (ABCB1) and breast cancer resistance protein (ABCG2) interplay in drug transport across the blood-brain barrier. Mol Pharm 2011;8:571-82. DOI PubMed

36. Pastan I, Gottesman MM, Ueda K, Lovelace E, Rutherford AV, Willingham MC. A retrovirus carrying an MDR1 cDNA confers multidrug resistance and polarized expression of P-glycoprtein in MDCK cells. ProcNatlAcadSciUSA 1988;85:4486-90. DOI PubMed PMC

37. Fung KL, Kapoor K, Pixley JN, et al. Using the BacMam baculovirus system to study expression and function of recombinant efflux drug transporters in polarized epithelial cell monolayers. Drug Metab Dispos 2016;44:180-8. DOI PubMed PMC

38. Elbakary B, Badhan RKS. A dynamic perfusion based blood-brain barrier model for cytotoxicity testing and drug permeation. Sci Rep 2020;10:3788. DOI PubMed PMC

39. Bhalerao A, Sivandzade F, Archie SR, Chowdhury EA, Noorani B, Cucullo L. In vitro modeling of the neurovascular unit: advances in the field. Fluids Barriers CNS 2020;17:22. DOI PubMed PMC

40. Helms HC, Abbott NJ, Burek M, et al. In vitro models of the blood-brain barrier: An overview of commonly used brain endothelial cell culture models and guidelines for their use. J Cereb Blood Flow Metab 2016;36:862-90. DOI PubMed PMC

41. O'Brown NM, Pfau SJ, Gu C. Bridging barriers: a comparative look at the blood-brain barrier across organisms. Genes Dev 2018;32:466-78. DOI PubMed PMC

42. Li W, Sparidans RW, Wang Y, Lebre MC, Beijnen JH, Schinkel AH. P-glycoprotein and breast cancer resistance protein restrict brigatinib brain accumulation and toxicity, and, alongside CYP3A, limit its oral availability. Pharmacol Res 2018;137:47-55. DOI PubMed

43. Li Y, Chen T, Miao X, et al. Zebrafish: A promising in vivo model for assessing the delivery of natural products, fluorescence dyes and drugs across the blood-brain barrier. Pharmacol Res 2017;125:246-57. DOI PubMed

44. Umans RA, Taylor MR. Zebrafish as a model to study drug transporters at the blood-brain barrier. Clin Pharmacol Ther 2012;92:56770. DOI PubMed PMC

45. Susaki EA, Tainaka K, Perrin D, Yukinaga H, Kuno A, Ueda HR. Advanced CUBIC protocols for whole-brain and whole-body clearing and imaging. Nat Protoc 2015;10:1709-27. DOI PubMed

46. Wassie AT, Zhao Y, Boyden ES. Expansion microscopy: principles and uses in biological research. Nat Methods 2019;16:33-41. DOI PubMed PMC

47. Umans RA, Henson HE, Mu F, et al. CNS angiogenesis and barriergenesis occur simultaneously. Dev Biol 2017;425:101-8. DOI PubMed PMC

48. Jeong JY, Kwon HB, Ahn JC, et al. Functional and developmental analysis of the blood-brain barrier in zebrafish. Brain Res Bull 2008;75:619-28. DOI PubMed

49. Fleming A, Diekmann H, Goldsmith P. Functional characterisation of the maturation of the blood-brain barrier in larval zebrafish. PLoS One 2013;8:e77548. DOI PubMed PMC

50. Quinonez-Silvero C, Hubner K, Herzog W. Development of the brain vasculature and the blood-brain barrier in zebrafish. Dev Biol 2020;457:181-90. DOI PubMed

51. van Leeuwen LM, Evans RJ, Jim KK, et al. A transgenic zebrafish model for the in vivo study of the blood and choroid plexus brain barriers using claudin 5. Biol Open 2018;7:bio030494. DOI PubMed PMC 
52. Loh YH, Christoffels A, Brenner S, Hunziker W, Venkatesh B. Extensive expansion of the claudin gene family in the teleost fish, Fugu rubripes. Genome Res 2004;14:1248-57. DOI PubMed PMC

53. Kiener TK, Sleptsova-Friedrich I, Hunziker W. Identification, tissue distribution and developmental expression of tjp1/zo-1, tjp2/zo-2 and tjp3/zo-3 in the zebrafish, Danio rerio. Gene Expr Patterns 2007;7:767-76. DOI PubMed

54. O'Brown NM, Megason SG, Gu C. Suppression of transcytosis regulates zebrafish blood-brain barrier function. Elife 2019;8:e47326. DOI PubMed PMC

55. Ben-Zvi A, Lacoste B, Kur E, et al. Mfsd2a is critical for the formation and function of the blood-brain barrier. Nature 2014;509:50711. DOI PubMed PMC

56. Guemez-Gamboa A, Nguyen LN, Yang H, et al. Inactivating mutations in MFSD2A, required for omega-3 fatty acid transport in brain, cause a lethal microcephaly syndrome. Nat Genet 2015;47:809-13. DOI PubMed PMC

57. Wang Y, Pan L, Moens CB, Appel B. Notch3 establishes brain vascular integrity by regulating pericyte number. Development 2014;141:307-17. DOI PubMed PMC

58. Ando K, Fukuhara S, Izumi N, et al. Clarification of mural cell coverage of vascular endothelial cells by live imaging of zebrafish. Development 2016;143:1328-39. DOI PubMed PMC

59. Korn J, Christ B, Kurz H. Neuroectodermal origin of brain pericytes and vascular smooth muscle cells. J Comp Neurol 2002;442:7888. DOI PubMed

60. Chen J, Poskanzer KE, Freeman MR, Monk KR. Live-imaging of astrocyte morphogenesis and function in zebrafish neural circuits. Nat Neurosci 2020;23:1297-306. DOI PubMed PMC

61. Jurisch-Yaksi N, Yaksi E, Kizil C. Radial glia in the zebrafish brain: functional, structural, and physiological comparison with the mammalian glia. Glia 2020;68:2451-70. DOI PubMed

62. Rowitch DH, Kriegstein AR. Developmental genetics of vertebrate glial-cell specification. Nature 2010;468:214-22. DOI PubMed

63. Grupp L, Wolburg H, Mack AF. Astroglial structures in the zebrafish brain. J Comp Neurol 2010;518:4277-87. DOI PubMed

64. Di Castro MA, Chuquet J, Liaudet N, et al. Local Ca2+ detection and modulation of synaptic release by astrocytes. Nat Neurosci 2011;14:1276-84. DOI PubMed

65. Neve LD, Savage AA, Koke JR, Garcia DM. Activating transcription factor 3 and reactive astrocytes following optic nerve injury in zebrafish. Comp Biochem Physiol C Toxicol Pharmacol 2012;155:213-8. DOI PubMed

66. Fischer S, Kluver N, Burkhardt-Medicke K, et al. Abcb4 acts as multixenobiotic transporter and active barrier against chemical uptake in zebrafish (Danio rerio) embryos. BMC Biol 2013;11:69. DOI PubMed PMC

67. Georges E, Bradley G, Garipey J, Ling V. Detection of P-glycoprotein isoforms by gene-specific monoclonal antibodies. Proc Natl Acad Sci USA 1990;87:152-6. DOI PubMed PMC

68. Park D, Haldi M, Seng W. Zebrafish: a new in vivo model for identifying P-glycoprotein efflux modulators. In: McGrath P, editor. Zebrafish: Methods for Assessing Drug Safety and Toxicity. 2011. p. 177-90.

69. Bard SM, Gadbois S. Assessing neuroprotective P-glycoprotein activity at the blood-brain barrier in killifish (Fundulus heteroclitus) using behavioural profiles. Mar Environ Res 2007;64:679-82. DOI PubMed

70. Kennedy CJ, Tierney KB, Mittelstadt M. Inhibition of P-glycoprotein in the blood-brain barrier alters avermectin neurotoxicity and swimming performance in rainbow trout. Aquat Toxicol 2014;146:176-85. DOI PubMed

71. Luckenbach T, Fischer S, Sturm A. Current advances on ABC drug transporters in fish. Comp Biochem Physiol C Toxicol Pharmacol 2014;165:28-52. DOI PubMed

72. Miller DS, Graeff C, Droulle L, Fricker S, Fricker G. Xenobiotic efflux pumps in isolated fish brain capillaries. Am J Physiol Regul Integr Comp Physiol 2002;282:R191-8. DOI PubMed

73. Annilo T, Chen ZQ, Shulenin S, et al. Evolution of the vertebrate ABC gene family: analysis of gene birth and death. Genomics 2006;88:1-11. DOI PubMed

74. Kobayashi I, Saito K, Moritomo T, Araki K, Takizawa F, Nakanishi T. Characterization and localization of side population (SP) cells in zebrafish kidney hematopoietic tissue. Blood 2008;111:1131-7. DOI PubMed

75. Long Y, Li Q, Li J, Cui Z. Molecular analysis, developmental function and heavy metal-induced expression of ABCC5 in zebrafish. Comp Biochem Physiol B Biochem Mol Biol 2011;158:46-55. DOI PubMed

76. Lu X, Long Y, Lin L, Sun R, Zhong S, Cui Z. Characterization of zebrafish Abcc4 as an efflux transporter of organochlorine pesticides. PLoS One 2014;9:e111664. DOI PubMed PMC

77. Kim SS, Im SH, Yang JY, et al. Zebrafish as a screening model for testing the permeability of blood-brain barrier to small molecules. Zebrafish 2017;14:322-30. DOI PubMed

78. Zoghbi SS, Liow JS, Yasuno F, et al. 11C-loperamide and its N-desmethyl radiometabolite are avid substrates for brain permeabilityglycoprotein efflux. J Nucl Med 2008;49:649-56. DOI PubMed

79. Bieczynski F, Burkhardt-Medicke K, Luquet CM, Scholz S, Luckenbach T. Chemical effects on dye efflux activity in live zebrafish embryos and on zebrafish Abcb4 ATPase activity. FEBS Lett 2020;595:828-43. DOI PubMed

80. Lu X, Long Y, Sun R, Zhou B, Lin L, et al. Zebrafish Abcb4 is a potential efflux transporter of microcystin-LR. Comp Biochem Physiol C Toxicol Pharmacol 2015;167:35-42. DOI PubMed

81. Lee TD, Lee OW, Brimacombe KR, et al. A high-throughput screen of a library of therapeutics identifies cytotoxic substrates of Pglycoprotein. Mol Pharmacol 2019;96:629-40. DOI PubMed PMC

82. Robey RW, Robinson AN, Ali-Rahmani F, et al. Characterization and tissue localization of zebrafish homologs of the human ABCB1 multidrug transporter. bioRxiv 2021. DOI

83. Gao Y, Zhang T, Terai H, et al. Overcoming resistance to the THZ series of covalent transcriptional CDK inhibitors. Cell Chem Biol 
2018;25:135-42.e5. DOI PubMed PMC

84. Yamasaki Y, Kobayashi K, Okuya F, et al. Characterization of P-glycoprotein humanized mice generated by chromosome engineering technology: its utility for prediction of drug distribution to the brain in humans. Drug Metab Dispos 2018;46:1756-66. DOI PubMed 